\title{
Primary Intraosseous Carcinoma of the Mandible: A Case Report
}

Badja $\mathrm{S}^{1^{*}}$, Kamal $\mathrm{D}^{1}$, Fadel $\mathrm{R}^{1}$, El alami Elamine $\mathrm{MN}^{1}$

${ }^{1}$ Departement of Maxillo-facial Surgery, ENT and CCF, OMAR IDRISSI Hospital, Chu Hassan Ii Fez, Morocco
${ }^{1}$ Departement of Maxillo-facial Surgery, ENT and CCF, OMAR IDRISSI Hospital, CHU Hassan Ii Fez, Morocco

DOI: $\underline{10.36347 / \text { sasjs.2021.v07i05.003 }}$

| Received: 26.02.2021 | Accepted: 12.04.2021 | Published: 05.05.2021

*Corresponding author: Badja $S$

Abstract

Primary intraosseous carcinoma (PIOC) is a rare tumor that has been infrequently reported. Carcinoma arising primarily from the jaw is a locally aggressive lesion with poor prognosis. Primary intraosseous carcinoma (PIOC) lesion develops either de novo remnants of odontogenic epithelium, odontogenic cyst/tumor, epithelium remnants, or/and salivary gland residues. This article describe a case of primary intraosseous carcinoma of mandible.

Keywords: Carcinoma, jaw neoplasms, malignant neoplasms, primary intraosseous carcinoma.

Copyright $\odot 2021$ The Author(s): This is an open-access article distributed under the terms of the Creative Commons Attribution 4.0 International License (CC BY-NC 4.0) which permits unrestricted use, distribution, and reproduction in any medium for non-commercial use provided the original author and source are credited.

\section{INTRODUCTION}

Primary intraosseous carcinoma of the jaw is a rare malignant lesion ranging up to $6 \%$ of all malignant neoplasms of maxillofacial région $[1,2]$. According to World Health Organization (WHO), International Histologic Classification of Tumors, Primary intraosseous carcinoma (PIOC) is a squamous cell carcinoma arising within the jaw that has no original connection with the surface epithelium of the oral mucosa, overlying skin, and antral or nasal mucosa developing from odontogenic epithelium or from a odontogenic cyst/tumor [3-5]. This article presents a case of PIOC with a discussion of the current perspectives.

\section{CASE REPORT}

A 64-year-old morrocan woman presented to the outpatient clinic with a complaint of a diffuse dull ache in the mandible since 6 months. Examination revealed no abnormalities other than excessive and painful mobility, of mandibular incisor tooth without displacement. No ulceration of the mucosa was observed and there was no initial connection with the oral mucosa or overlying skin [Figure1], the regional lymphadenopathy was absent. The CT scan showed a total lysis of mandibular symphysis (Figure 2). Incisional biopsy revealed proliferative stratified squamous epithelium with dysplastic squamous islands exhibiting features of hyperchromatism, pleomorphism, and individual cell keratinization. The tumor cells showed no contact with the normal appearing overlying mucosa. Based on all findings, a final diagnosis of Primary Intraosseous Carcinoma of the mandible was given.

The patient underwent an interruptive pelvimandibulectomy and functional cervical lymph node excision. Reconstruction was performed with free fibula graft [Figure 3]. Post operative sequelae were marked by necrosis of the flap by arterial thrombosis. A surgical revision with removal of the flap, insertion of a space maintaining plate and covering with a right pectoral and left naso-labial flap. After surgery, the patient underwent radiotherapie to prevent the likelihood of metastasis and poor outcome. Patient is under periodic followup till date without any evidence of malignancy.

A two years follow up showed a good evolution and no sign of recurrence. 


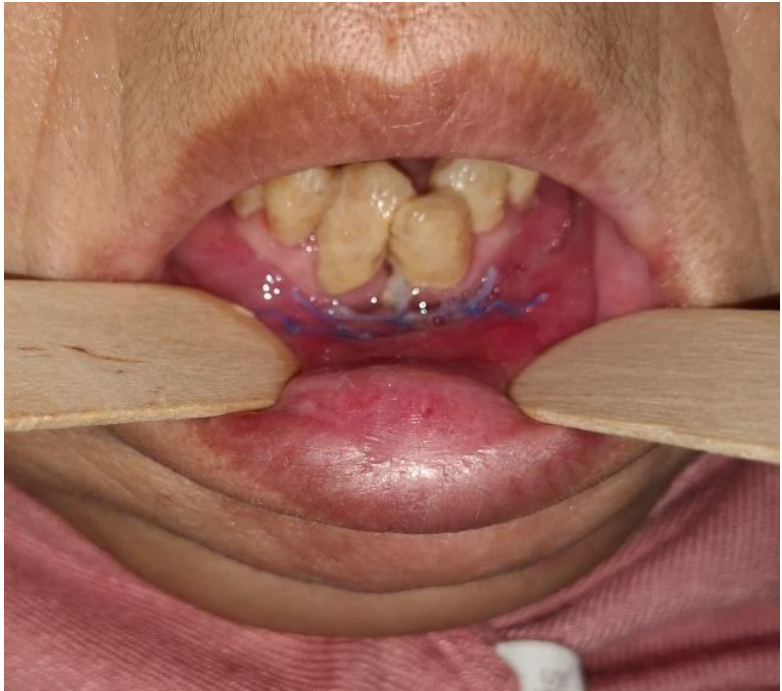

Fig-1: Intra-oral image after bone biopsy by vestibular approach. Note the intact overlying alveolar mucosa sutured with vicryl 3-0

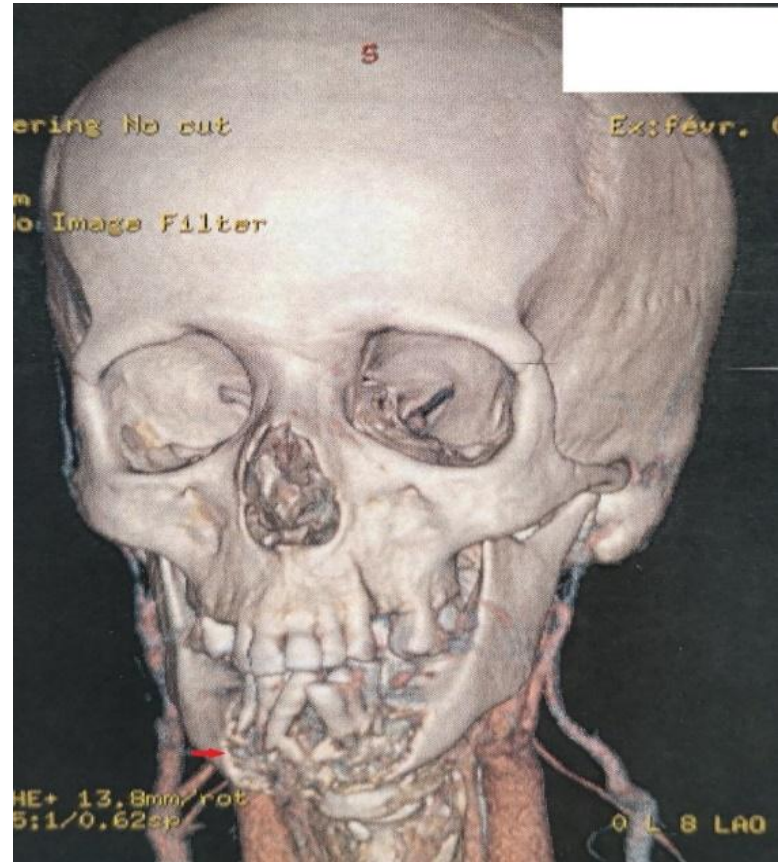

Fig-2: CT scan showed a total lysis of mandibular symphysis

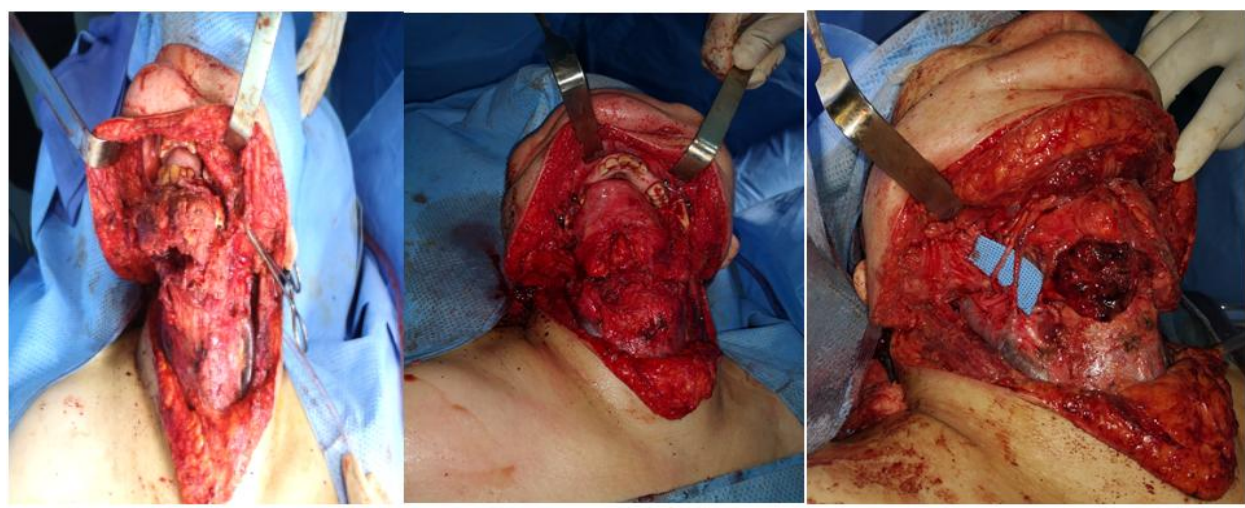

Fig-3: Peroperative aspects of different surgical times, from left to right: 1- Exposure of the mandibular tumour by external route of PAUL ANDRE. 2- Tumor resection in monobloc carrying the anterior portion of the mandible and the oral floor. 3Arterial and venous microsurgical anastomosis of the free osteomyocutane flap of the fibula 


\section{DISCUSSION}

Primary intraosseous carcinoma is an uncommon neoplasm. The WHO defines PIOC of the jaw as a "squamous cell carcinoma arising within the jaw, having no initial connection with the oral mucosa and presumably developing from residues of the odontogenic epithelium." PIOC was subclassified into carcinoma arising de novo, carcinomas arising from ameloblastoma and carcinomas arising from odontogenic cyst. [4] It appeared logical to also include intraosseous mucoepidermoid carcinomas as a fourth type of PIOC [6]. The current classification is as follows:[10].

Type 1: PIOC ex-odontogenic cyst

Type 2A: Malignant ameloblastoma

Type 2B: Ameloblastic carcinoma arising de novo, exameloblastoma or ex-odontogenic cyst

Type 3: PIOC arising de novo: (a) Keratinizing type: (b) Non-keratinizing type

Type 4: Intraosseous mucoepidermoid carcinoma

The WHO's [11] histological classification mentions that PIOCs can develop from odontogenic epithelium. Lucas [8] presumed that this carcinoma could arise from odontogenic rests or from epithelium trapped within deeper structures during fusion of facial process. However, investigators now believe that embryonic tissues, except for the epithelial component of the incisive canal, have little or no pathogenic significance in the development of jaw cyst or tumors [9].

For a carcinoma to develop, presence of epithelium is a must, and it can include remnants of dental lamina, remnants of Hertwig's epithelial root sheath, remnants of enamel organ, odontogenic tumors or odontogenic cyst lining, entrapped epithelium during fusion of facial process and salivary gland inclusions [6]. The present tumor was not associated with any embedded or missing teeth. The pooled analysis of the world literature of PIOC[10] reveals that the mean age of the patients of PIOC is 52.3 years, with the age ranging from 4 years to 81 years, with $65 \%$ of the patients being in the sixth or seventh decades of life.

The tumor affects men more often than women, with a male:female ratio ranging from 1.5 to $3.5: 1$ [7, 10, 11,12]. Majority of the cases arise in the posterior mandible where remnants of the dental lamina are most likely to be the source of epithelium.

Although the clinical features of PIOCs are non-specific, Thomas et al. [10] have reported that principal manifestations of de novo PIOC cases were sensory disturbances like paraesthesia and numbness, mimicking facial neurological problems. The histologic features of PIOC are not pathognomonic and diagnosis is often difficult.
Additional criteria for categorizing a lesion as PIOC, such as: (a) intact oral mucosa before diagnosis; (b) microscopic evidence of squamous cell carcinoma without a cystic component or other odontogenic tumor cells; and (c) absence of another primary tumor on chest radiographs obtained at the time of diagnosis and during a follow-up period of more than 6 months, have been suggested by Suei et al.[14,15].

Treatment for PIOC is principally wide local resection and radiotherapy and chemotherapy may be performed as adjunctive treatments. Its prognosis is relatively poor and the 5-year survival rate ranges from $30 \%$ to $40 \%$ [7, 10], with a mean survival rate of 40.6 months if treated by radical surgery with radiotherapy [13]. PIOC is occasionally, but not always, associated with metastasis to the regional lymph nodes [15]. In this case, no regional lymph node enlargement was observed at the initial examination. In this case, the patient underwent a interruptive pelvimandibulectomy and Functional cervical lymph node.

\section{CONCLUSION}

The purpose of reporting this case is to add to the existing database about this rare tumor, which will further help in an establishment of theories about the origin and biological behavior of these truly uncommon neoplasms.

\section{REFERENCES}

1. Aboul-Hons Centenero S, Marí Roig A, Piulachs Clapera P, Juárez Escalona I, Monner Diéguez A, Díaz Carandell A, Lluch JM, Pericot Ayats J. Primary Intraosseous Carcinoma and Odontogenic Cyst: Three new cases and review of the Literature.

2. Hayashido Y, Yoshioka Y, Shintani T, Hamana T, Koizumi KI, Ishida Y, Okamoto T. Primary intraosseous carcinoma of mandible associated with elevation of serum carcinoembryonic antigen level. Oral Oncology Extra. 2005 Nov 1;41(10):267-71.

3. Reddy PB, Reddy BS, Prasad N. An unusual case report of primary intraosseous carcinoma impersonating as missing mandible. International Journal of Oral and Maxillofacial Pathology. 2012;3(3):41-44.

4. Kramer IR, Pindborg JJ, Shear M. Histological typing of odontogenic tumors. 2nd ed. Berlin: Springer-Verlag; $1992: 25$.

5. Huang JW, Luo HY, Li Q, Li TJ. Primary intraosseous squamous cell carcinoma of the jaws: Clinicopathologic presentation and prognostic factors. Archives of pathology \& laboratory medicine. 2009 Nov;133(11):1834-40.

6. Waldron CA, Mustoe TA. Primary intraosseous carcinoma of the mandible with probable origin in an odontogenic cyst. Oral surgery, oral medicine, oral pathology. 1989 Jun 1;67(6):716-24.

7. To EH, Brown JS, Ward-Booth RP, Avery BS. Primary intraosseous carcinoma of the jaws. Three 
new cases and a review of the literature. British Journal of Oral and Maxillofacial Surgery. 1991 Feb 1;29(1):19-25.

8. Lucas RB. Pathology of tumors of the oral tissues. 4th ed. London: Churchill Livingstone; $1984: 140-1$.

9. Spouge JD. Odontogenic tumors: A unitarian concept. Oral Surg Oral Med Oral Pathol. 1967;24:392-403.

10. Thomas G, Pandey M, Mathew A, Abraham EK, Francis A, Somanathan T, Iype EM, Sebastian P, Nair MK. Primary intraosseous carcinoma of the jaw: pooled analysis of world literature and report of two new cases. International journal of oral and maxillofacial surgery. 2001 Aug 1;30(4):349-55.

11. Elzay RP. Primary intraosseous carcinoma of the jaws: review and update of odontogenic carcinomas. Oral Surgery, Oral Medicine, Oral Pathology. 1982 Sep 1;54(3):299-303.

12. Jethlia A, Keluskar V, Shetti A. Primary intraosseous carcinoma of mandible. J Indian Acad Oral Med Radiol. 2009;21:122-5.

13. Zwetyenga N, Pinsolle J, Rivel J, MajoufreLefebvre C, Faucher A, Pinsolle V. Primary intraosseous carcinoma of the jaws. Arch Otolaryngol Head Neck Surg. 2001;127:794-7.

14. Vander Waal RI, Buter J, van der Waal I. Oral metastases: Report of 24 cases. $\mathrm{Br} \mathrm{J}$ Oral Maxillofac Surg. 2003;41:3-6.

15. Suei Y, Tanimoto K, Taguchi A, Wada T. Primary intraosseous carcinoma: Review of the literature and diagnostic criteria. J Oral Maxillofac Surg. 1994;52:580-3. 